\title{
THE PROPAGATION OF ERROR IN LINEAR PROBLEMS
}

BY

\section{A. T. LONSETH}

The application of mathematics to natural phenomena often brings up the question: when a linear vector equation is changed slightly, how much of a change results in the solution? Here "vector" is used to include, for example, vectors in Hilbert space and elements of (linear) function spaces; also it is assumed $\left.{ }^{1}\right)$ that the equation is uniquely solvable. We formulate and answer the question in $\$ 2$ for a linear equation in a Banach space, then specialize to deduce perturbation limits for linear algebraic systems, infinite systems, and integral equations. Finally we obtain error limitations for certain approximate methods of solving infinite linear systems (method of segments) and integral equations (method of Goursat-Schmidt). It is hoped that these limitations may be useful to applied mathematicians.

The methods and results of this paper unify and extend investigations concerning algebraic systems by F. R. Moulton [15](2), Etherington [6] and the author $[13,14]$; and on integral equations of Fredholm type and second kind by Tricomi [21]. However, they do not cover perturbation questions associated with characteristic values and characteristic functions, which have been studied by Lord Rayleigh [16, p. 115], Courant [4, p. 296] and Mrs. Adams [1].

$\$ 1$ is expository, containing as much about abstract vector spaces as is needed for $\$ 2$.

1. Vector spaces. It will be useful to collect here some facts about normed linear vector spaces (spaces $L$ ), Banach spaces (spaces $B$ ), and linear transformations. A space $L$ is linear: if vector $x \in L$ and $a$ is any complex number, the product $a x$ is defined and $a x \in L$; if also $y \in L$, the sum $x+y$ is defined and $x+y \in L$. With each element $x$ of $L$ is associated a non-negative real number $\|x\|$, its norm; $\|x\|>0$ unless $x=\Theta$, the zero-element of $L ;\|\Theta\|=0$. The norm has properties of an absolute value: $\|a x\|=|a| \cdot\|x\|,\|x+y\| \leqq\|x\|+\|y\|$. (We have described a complex space $L$; in a real space, number $a$ must be real.)

A normed linear vector space is a Banach space $B[2, \mathrm{p} .53]$ if it is furthermore complete: if $\left\{x_{n}\right\}$ is an infinite sequence of elements of $B$, and if $\left\|x_{m}-x_{n}\right\| \rightarrow 0$ as $m, n \rightarrow \infty$, there exists a vector $x_{0}$ of $B$ such that $\left\|x_{n}-x_{0}\right\| \rightarrow 0$ as $n \rightarrow \infty$ (strong completeness). Examples of such spaces are listed in Banach's book [2, pp. 10-12, examples 3-10], and several occur in the remainder of this paper.

Presented to the Society, November 26, 1943; received by the editors October 10, 1945.

(1) Uniqueness is not required in 887 and 12.

(2) Numbers in brackets refer to the list of references at the end of the paper. 
We shall be concerned with a transformation $T$ which carries each element $x$ of $L$ into an $x^{\prime}$ in $L: T x=x^{\prime}$. Such a $T$ will be said to be "in $L$." We suppose $T$ to be additive: $T(x+y)=T x+T y$. If further $T$ is continuous in the sense that $\left\|T x_{n}-T x_{0}\right\| \rightarrow 0$ as $\left\|x_{n}-x_{0}\right\| \rightarrow 0$ (strong continuity), it is linear, and there exists $[2$, p. 54] a real constant $M(T)$ such that whatever $x$ in $L$

$$
\|T x\| \leqq M(T)\|x\| .
$$

We suppose $M(T)$ to be the smallest constant satisfying (1.1), and call it the (precise) upper bound of $T\left(^{3}\right)$. Similarly we define the (precise) lower bound of $T$ as the largest constant $m(T)$ satisfying

$$
\|T x\| \geqq m(T)\|x\|,
$$

whatever $x$ in $L$. A linear $T$ is also homogeneous $[2$, p. 36].

If $T$ is reciprocally (1-1) and $m(T)>0$, the inverse $T^{-1}$ is linear [2, p. 145] and

$$
m(T) M\left(T^{-1}\right)=1 .
$$

To prove (1.3) we write $T x=x^{\prime}$, so $x=T^{-1} x^{\prime}$. Hence $\left\|x^{\prime}\right\| \geqq m(T)\|x\|$, in which $m(T)$ is the largest constant permissible over $L$. Therefore $1 / m(T)$ is the smallest constant $k$ satisfying $\|x\| \leqq k\left\|x^{\prime}\right\|$; but this defines $M\left(T^{-1}\right)$.

The sum $T_{1}+T_{2}$ of two additive transformations in $L$ is defined as usual to be the transformation which carries $x$ into $T_{1} x+T_{2} x$, and the product $T_{1} T_{2}$ as that which carries $x$ into $T_{1}\left(T_{2} x\right)$. Addition is both commutative and associative; multiplication is associative, and distributive over addition. Though multiplication is not in general commutative, it is so for powers of the same transformation. By definition, $T^{0}=I$, the "identity"; $m(I)=M(I)$ $=1$. If $T_{1}$ and $T_{2}$ are furthermore continuous, so are $T_{1}+T_{2}$ and $T_{1} T_{2}$ and

$$
\begin{gathered}
M\left(T_{1}+T_{2}\right) \leqq M\left(T_{1}\right)+M\left(T_{2}\right), \\
M\left(T_{1} T_{2}\right) \leqq M\left(T_{1}\right) M\left(T_{2}\right) .
\end{gathered}
$$

Most of what follows depends on solving an equation

$$
T x \equiv x-\mathrm{K} x=y
$$

for $x, y$ being given, and limiting $\|x\|$, under the assumption that $\mathrm{K}$ is linear and that

$$
M(\mathrm{~K})<1 .
$$

A unique solution is known to exist in each of the following two settings:

(a) $y \in B, a$ Banach space, and $x$ is sought in $B$;

(b) $y \in L$, a normed linear vector space, $\mathbf{K}$ is completely continuous over $L$, and $x$ is sought in $L$. In either case, the solution is representable as a Liouville-

$\left(^{(3)} M(T)\right.$ is also called the "norm" of $T$. 
Neumann-Hilb series

$$
x=\sum_{n=0}^{\infty} \mathrm{K}^{n} y
$$

and is limited by

$$
\|x\| \leqq\|y\| /\{1-M(\mathrm{~K})\} .
$$

Thus $T=I-\mathrm{K}$ has the unique inverse $T^{-1}=\sum_{n=0}^{\infty} \mathrm{K}^{n}$, and

$$
M\left(T^{-1}\right) \leqq 1 /\{1-M(\mathrm{~K})\}, \quad m(T) \geqq 1-M(\mathrm{~K}) .
$$

The idea underlying the proofs is that of iteration. If a solution $x$ of (1.6) were to exist, it would also satisfy

$$
\begin{aligned}
x & =y+\mathrm{K} y+\mathrm{K}^{2} y+\cdots+\mathrm{K}^{n} y+\mathrm{K}^{n+1} x \\
& =x_{n}+\mathrm{K}^{n+1} x .
\end{aligned}
$$

Since $x_{n+p}-x_{n}=\sum_{i=n+1}^{i=n+p} K^{i} y$, it follows from inequalities (1.4), (1.5) and (1.7) that $\left\|x_{n+p}-x_{n}\right\| \leqq M(\mathbf{K})^{n}\|y\| /\{1-M(\mathbf{K})\}$. Hence $\left\|x_{n+p}-x_{n}\right\| \rightarrow 0$ as $n \rightarrow \infty$, whatever the behavior of $p$. But in case (a), $B$ is complete and consequently contains an $x$ such that $\left\|x_{n}-x\right\| \rightarrow 0$-that is, (1.8). Inequality (1.9) is an immediate consequence, as is (1.6) [2, p. 159].

In case (b), the space is no longer complete. We observe that $x_{n}-y=\mathbf{K} x_{n-1}$ and that $\left\|x_{n-1}\right\| \leqq\|y\| /\{1-M(K)\}$. By definition [2, p. 96], the completely continuous transformation $K$ transforms a bounded set of vectors into a compact [2, p. 9] one; hence $\left\{x_{n}-y\right\}$ contains a subsequence $\left\{x_{n_{k}}-y\right\}$ which converges (strongly) to an element $x-y$.of $L$. Since also $\left\|x_{n_{k}}-x_{n}\right\| \rightarrow 0$ as $n_{k} \rightarrow \infty$ and $n \rightarrow \infty,\left\|x_{n}-x\right\| \rightarrow 0$.

In each case there is but one solution. For if also $x^{\prime}-\mathrm{K} x^{\prime}=y, x-x^{\prime}$ $=\mathrm{K}\left(x-x^{\prime}\right)$ and, by (1.7), $\left\|x-x^{\prime}\right\|<\left\|x-x^{\prime}\right\|$ unless $x-x^{\prime}=\Theta$, the zero element of $L$.

2. A general bound. If vector $y \in B$, a Banach space, and if $T$ is an additive transformation in $B$ with the (unique) linear inverse $T^{-1}$ in $B$, equation

$$
T x=y
$$

is solved by $x=T^{-1} y ; x \in B$, and

$$
\|x\| \leqq M\left(T^{-1}\right)\|y\| \text {. }
$$

If now $y$ is replaced by $y+\eta$, where $\eta \in B$, and $T$ by $T+\tau$, where $\tau$ is a linear transformation in $B$, the solution $x$ of (2.1) will change to the solution $x+\xi$ of

$$
(T+\tau)(x+\xi)=y+\eta .
$$

Because of (2.1), $T \xi+\tau \xi+\tau x=\eta$. This equation for $\xi$ can finally be written as 


$$
\xi+T^{-1} \tau \xi=T^{-1}(\eta-\tau x),
$$

which is of type (1.6), with $\mathrm{K}=-T^{-1} \tau$. Since both $T^{-1}$ and $\tau$ were assumed linear, $\mathrm{K}$ is bounded by (1.5). If now

$$
M\left(T^{-1} \tau\right)<1,
$$

equation (2.4) has a unique solution $\xi$ in $B$, with the representation (1.8),

$$
\xi=\sum_{n=0}^{\infty}\left(-T^{-1} \tau\right)^{n}\left(T^{-1} \eta-T^{-1} \tau x\right) .
$$

Inequality (1.9) also applies, so that, under the conditions stated, we can formulate our fundamental theorem.

THEOREM. If $M\left(T^{-1} \tau\right)<1$, there exists a unique solution $\xi$ in $B$ of $(T+\tau)$ $(x+\xi)=y+\eta$, where $T x=y$; and

$$
\begin{aligned}
\|\xi\| & \leqq\left\{\left\|T^{-1} y\right\|+\left\|T^{-1} \tau x\right\|\right\} /\left\{1-M\left(T^{-1} \tau\right)\right\} \\
& \leqq\left\{M\left(T^{-1}\right)\|\eta\|+M\left(T^{-1} \tau\right)\|x\|\right\} /\left\{1-M\left(T^{-1} \tau\right)\right\} .
\end{aligned}
$$

(It will be recalled that (2.2) limits $\|x\|$.)

The following is by (1.3) and (1.5) an obvious consequence.

Corollary. If $M(\tau)<m(T)$,

$$
\|\xi\| \leqq\{\|\eta\|+M(\tau)\|x\|\} /\{m(T)-M(\tau)\} .
$$

The foregoing discussion applies almost verbatim to an equation in a space $L$ which is not complete, provided that complete continuity is required of $\tau$. For if one of two linear transformations is completely continuous, so is their product; and we have hypotheses (b) of $\$ 1$ applying to (2.4).

Inequalities (2.6) and (2.7) are still true if $T^{-1}$ is not unique, if only by $x$ is meant the solution $T^{-1} y$. This observation will be used in $\$ \S 7$ and 12 .

3. Perturbation of the inverse. An alternative form of the problem solved in $\$ 2$ is: by how much does the inverse $(T+\tau)^{-1}$ of $T+\tau$ differ from $T^{-1}$ ? For simplicity, we take the existence and uniqueness of the inverses for granted. We write

$$
(T+\tau)^{-1}=T^{-1}+\sigma,
$$

from which

$$
\begin{aligned}
I & =(T+\tau) T^{-1}+(T+\tau) \sigma \\
& =I+\tau T^{-1}+T \sigma+\tau \sigma
\end{aligned}
$$

and

$$
\sigma=-T^{-1} \tau T^{-1}-T^{-1} \tau \sigma,
$$


so that

$$
M(\sigma) \leqq M\left(T^{-1} \tau T^{-1}\right)+M\left(T^{-1} \tau\right) M(\sigma) .
$$

Consequently, if $M\left(T^{-1} \tau\right)<1$,

$$
M(\sigma) \leqq M\left(T^{-1} \tau T^{-1}\right) /\left\{1-M\left(T^{-1} \tau\right)\right\} .
$$

Equation (2.3) has for its solution

$$
\begin{aligned}
x+\xi & =(T+\tau)^{-1}(y+\eta) \\
& =\left(T^{-1}+\sigma\right)(y+\eta) ;
\end{aligned}
$$

or, since $x=T^{-1} y$,

$$
\xi=T^{-1} \eta+\sigma y+\sigma \eta \text {. }
$$

From (3.3) and (3.2) can be derived the inequality

$$
\|\xi\| \leqq M\left(T^{-1}\right)\left\{\|\eta\|+M\left(T^{-1} \tau\right)\|y\|\right\} /\left\{1-M\left(T^{-1} \tau\right)\right\},
$$

which also results from (2.6) and (2.2).

4. The algebraic case. Now $x$ is an ordinary $n$-dimensional vector with complex components $\left(x_{1}, x_{2}, \cdots, x_{n}\right)$; the matter of a norm we shall take up later. Transformation $T$ is a homogeneous linear transformation of matrix $A=\left(a_{i j}\right)$, and $T x=y$ becomes

$$
\sum_{j=1}^{n} a_{i j} x_{j}=y_{i}, \quad i=1,2, \cdots, n,
$$

where $a_{i j}$ and $y_{i}$ are complex numbers. Existence of the inverse $T^{-1}=A^{-1}$ is equivalent to nonvanishing of the determinant $\Delta$ of $A$; if $\Delta \neq 0, A^{-1}=\left(b_{i j}\right)$ $=\left(A_{j i} / \Delta\right)$, where $A_{i j}$ is the cofactor of $a_{i j}$ in $\Delta$. We write $\alpha=\left(\alpha_{i j}\right)$ for the matrix of error-transformation $\tau$. Then the perturbed system is

$$
\sum_{j=1}^{n}\left(a_{i j}+\alpha_{i j}\right)\left(x_{j}+\xi_{j}\right)=y_{i}+\eta_{i}, \quad i=1,2, \cdots, n .
$$

The resulting system for $\xi$ is

$$
\xi_{i}=\sum_{j=1}^{n} b_{i j} \eta_{j}-\sum_{j=1}^{n} \sum_{k=1}^{n} b_{i j} \alpha_{j k} x_{k}-\sum_{j=1}^{n} \sum_{k=1}^{n} b_{i j} \alpha_{j k} \xi_{k}, \quad i=1,2, \cdots, n .
$$

Application of the results of $\$ 2$ depends on the introduction of a suitable norm $\|x\|$. From among the various possibilities we select two for detailed treatment, namely $\sum_{i=1}^{i=n}\left|x_{i}\right|$ and $\left(\sum_{i=1}^{i=n}\left|x_{i}\right|^{2}\right)^{1 / 2}$. The details can be worked out just as easily for $\|x\|=\left(\sum_{i=1}^{i=n}\left|x_{i}\right|^{p}\right)^{1 / p}, p>1$, with the help of the inequalities of Hölder and Minkowski. Another possibility is $\|x\|=\max \left\{\left|x_{i}\right|\right\}$. With each of these norms the space is complete and a linear transformation is completely continuous: the theory in $\S \S 1$ and 2 is embarrassingly adequate. 
$1^{\circ}$. The norm $\|x\|=\sum_{i=1}^{i=n}\left|x_{i}\right|$. Inequality (2.6) requires knowledge of $M\left(T^{-1} \tau\right)$, or of an upper bound for $M\left(T^{-1} \tau\right)$. Here $T^{-1} \tau$ is the matrix product $A^{-1} \alpha=\left(b_{i j}\right)\left(\alpha_{i j}\right)=\left(\sum_{i=1}^{i=n} b_{i k} \alpha_{k j}\right)$. If each $\left|\alpha_{i j}\right| \leqq \delta$,

$$
\begin{aligned}
\left\|A^{-1} \alpha x\right\| & =\sum_{i=1}^{n}\left|\sum_{j=1}^{n} \sum_{k=1}^{n} b_{i j} \alpha_{j k} x_{k}\right| \\
& \leqq \delta\|x\| \sum_{i=1}^{n} \sum_{j=1}^{n}\left|b_{i j}\right|,
\end{aligned}
$$

so that

$$
M\left(A^{-1} \alpha\right) \leqq \delta \sum_{i=1}^{n} \sum_{j=1}^{n}\left|b_{i j}\right|
$$

Consequently (2.6) applies if

$$
\delta<|\Delta| / \sum_{i=1}^{n} \sum_{j=1}^{n}\left|A_{i j}\right|
$$

If also each $\left|\eta_{i}\right|<\delta$, the first inequality of (2.6) gives

$$
\|\xi\| \leqq \frac{\delta}{|\Delta|} \sum_{i=1}^{n} \sum_{j=1}^{n}\left|A_{i j}\right|\{1+\|x\|\} /\left\{1-\frac{\delta}{|\Delta|} \sum_{i=1}^{n} \sum_{j=1}^{n}\left|A_{i j}\right|\right\}
$$

where - to repeat- $\Delta$ is the nonvanishing determinant of system (4.1), $A_{i j}$ is the cofactor of $a_{i j}$ in $\Delta$, each $\left|\alpha_{i j}\right|$ and $\left|\eta_{i}\right|$ in (4.2) is no greater than $\delta$, and $\delta$ satisfies (4.4).

Since each $\left|\xi_{i}\right| \leqq\|\xi\|$, inequality (4.5) also limits the error in each component $x_{i}$ of $x$. However, a more genuine limitation can be found. For, taking absolute values in (4.3) we get

$$
\left|\xi_{i}\right| \leqq \delta \sum_{j=1}^{n}\left|b_{i j}\right|\{1+\|x\|+\|\xi\|\}, \quad i=1,2, \cdots, n,
$$

which, when combined with $(4.5)$, yields the inequality $[13$, p. 335]

$$
\begin{array}{r}
\left|\xi_{i}\right| \leqq \frac{\delta}{|\Delta|} \sum_{j=1}^{n}\left|A_{j i}\right|\left\{1+\sum_{j=1}^{n}\left|x_{j}\right|\right\} /\left\{1-\frac{\delta}{|\Delta|} \sum_{j=1}^{n} \sum_{k=1}^{n}\left|A_{j k}\right|\right\}, \\
i=1,2, \cdots, n .
\end{array}
$$

As was pointed out in [13], ratio

$$
\sum_{i=1}^{n} \sum_{j=1}^{n}\left|A_{i j}\right| /|\Delta|=\sum_{i=1}^{n} \sum_{j=1}^{n}\left|b_{i j}\right|
$$

measures the sensitivity of (4.1) to errors in the coefficients. In $\$ 5$ we shall present a geometrical analysis of this sensitivity. 
$2^{\circ}$. The norm $\|x\|=\left(\sum_{i=1}^{i=n}\left|x_{i}\right|^{2}\right)^{1 / 2}$. As above, we need an upper bound for $M\left(A^{-1} \alpha\right)$. Since now

$$
\left\|A^{-1} \alpha x\right\|^{2}=\sum_{i=1}^{n}\left|\sum_{j=1}^{n} \sum_{k=1}^{n} b_{i j} \alpha_{j k} x_{k}\right|^{2},
$$

two applications of the Lagrange-Cauchy inequality show that

$$
\begin{aligned}
M\left(A^{-1} \alpha\right) & \leqq M\left(A^{-1}\right) M(\alpha) \\
& \leqq\left(\sum_{i=1}^{n} \sum_{j=1}^{n}\left|b_{i j}\right|^{2}\right)^{1 / 2}\left(\sum_{i=1}^{n} \sum_{j=1}^{n}\left|\alpha_{i j}\right|^{2}\right)^{1 / 2} .
\end{aligned}
$$

Consequently, if

$$
\sum_{i=1}^{n} \sum_{j=1}^{n}\left|\alpha_{i j}\right|^{2}<|\Delta|^{2} / \sum_{i=1}^{n} \sum_{j=1}^{n}\left|A_{i j}\right|^{2}
$$

inequality (2.7) holds, with $m(T)$ replaced by $|\Delta| /\left(\sum \sum\left|A_{i j}\right|^{2}\right)^{1 / 2}$ and $M(\tau)$ by $\left(\sum \sum\left|\alpha_{i j}\right|^{2}\right)^{1 / 2}$. Thus we limit $\|\xi\|$. From (4.3) we also get a bound for $\left|\xi_{i}\right|:$

$$
\begin{aligned}
\left|\xi_{i}\right| \leqq\left(\sum_{j=1}^{n}\left|b_{i j}\right|^{2}\right)^{1 / 2}\left\{\|\eta\|+\left(\sum_{j=1}^{n} \sum_{k=1}^{n}\left|\alpha_{j k}\right|^{2}\right)^{1 / 2}(\|x\|+\|\xi\|)\right\} & \\
i & =1,2, \cdots, n .
\end{aligned}
$$

All such inequalities, whatever the norm, assume that the inverse matrix $A^{-1}$ is known. However( $\left.{ }^{4}\right)$, if

$$
\left|a_{i i}\right|>\sum_{k=1}^{i-1}\left|a_{i k}\right|+\sum_{k=i+1}^{n}\left|a_{i k}\right|
$$

it is possible to compute an upper bound for $M\left(A^{-1}\right)$ without computing $A^{-1}$ itself; for an inequality of $\mathrm{H}$. von Koch $[5, \mathrm{p} .125]$ supplies a lower bound to $|\Delta|$, and the minors $A_{i j}$ can be limited by Hadamard's determinant inequality.

Direct computation of $A^{-1}$ is usually laborious. Hotelling [9, p. 14] describes an iterative method which converges very rapidly, and derives for it a usable limit of accuracy. This limit is somewhat improved in [10].

Special interest attaches to the case in which $A$ is the real, symmetric matrix of a positive definite quadratic form. Then, with $\|x\|=\left(\sum_{i=1}^{i=n}\left|x_{i}\right|^{2}\right)^{1 / 2}$,

$$
\lambda_{1}\|x\| \leqq\|A x\| \leqq \lambda_{n}\|x\|,
$$

where $\lambda_{1}$ and $\lambda_{n}$ are respectively the smallest and the largest roots of the characteristic equation

(4) For this remark I am indebted to H. T. Davis. 


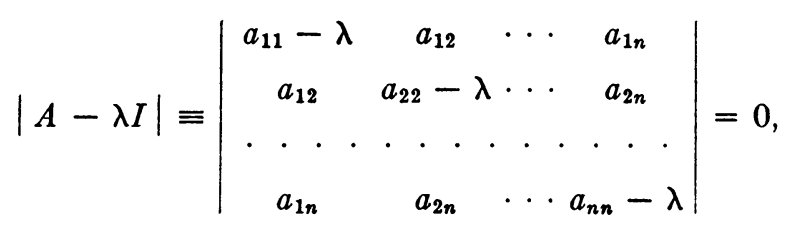

all of which are real and positive. Since the equality signs in (4.9) are actually realized for particular vectors, $m(A)=\lambda_{1}$ and $M(A)=\lambda_{n}$. Thus $M\left(A^{-1}\right)$ $=1 / m(A)=\lambda_{1}{ }^{-1}$. If considerable precision is required in $2^{\circ}$ above, $A^{-1}$ may be computed and its greatest characteristic root $M\left(A^{-1}\right)$ approximated as in $[9$, p. 28]. Synge [20] has suggested a modification of the "relaxation" method which may be useful for computing the inverse in the positive definite case, perhaps especially to furnish a starter for Hotelling's process. Finally, it should be mentioned that any linear system can be made symmetric $[9, \mathrm{p} .11]$, and, if $\Delta \neq 0$, positive definite.

5. Geometry of the algebraic problem. When the $a$ 's and $y$ 's are real, equations (4.1) represent a system of $n(n-1)$-flats in a real $n$-space; equations (4.2) represent $n$ neighboring $(n-1)$-flats. Geometrically, the problem of $\$ 4$ was to bound the "distance" between the intersection of $(4.1)$ and that of (4.2).

If $n=2$, angle $\theta$ between the lines (4.1) is given by

$$
\sin \theta=\frac{|\Delta|}{\left(a_{11}^{2}+a_{12}^{2}\right)^{1 / 2}\left(a_{21}^{2}+a_{22}\right)^{1 / 2}} .
$$

If $\sin \theta$ is small-that is, if determinant $\Delta$ is small relative to its elementsthe lines are nearly parallel, and small changes in the coefficients may produce a large displacement of the intersection point.

If $n=3$, three situations make for sensitivity: (i) the planes (4.1) may be nearly parallel; (ii) two of them may be nearly parallel, the third not; (iii) the three may be nearly coaxial. Each case corresponds to smallness of volume of a tetrahedron, formed as follows: about the intersection point of the three planes as center describe the unit sphere; the positive half rays of the lines of intersection of the planes intersect this sphere in three points which, with the center, are the vertices of the tetrahedron. Its volume is

$$
V=\frac{1}{3 !} \frac{|\Delta|^{2}}{\left(\prod_{i=1}^{8} \sum_{j=1}^{8} A_{i j}^{2}\right)^{1 / 2}},
$$

in our customary notation.

If $n>3$, each $n-1$ of the $n$ hyperplanes (4.1) intersect in a line, and these $n$ lines all pass through the intersection of (4.1). This point, plus the $n$ points in which the positive half-rays intersect the unit sphere about it, form an $n$-dimensional simplex the size of whose volume 


$$
V=\frac{1}{n !} \frac{|\Delta|^{n-1}}{\left(\prod_{i=1}^{n} \sum_{j=1}^{n} A_{i j}^{2}\right)^{1 / 2}}
$$

may be taken as a criterion of sensitivity in the general case.

As was also seen in $\S 4$, small $\Delta$ and large $A_{i j}$ 's cause sensitivity to error. It is remarkable that the denominator in (5.3) is an upper bound $\max \left|\Delta^{*}\right|$ of the adjoint $\Delta^{*}$ of $\Delta$, as supplied by Hadamard's inequality. In fact,

$$
V=\frac{1}{n !} \frac{\left|\Delta^{*}\right|}{\max \left|\Delta^{*}\right|}=\frac{1}{n !} \frac{\left|\Delta^{-1}\right|}{\max \left|\Delta^{-1}\right|},
$$

and we conclude that the more nearly the determinant $\Delta^{-1}$ of inverse matrix $A^{-1}$ approaches its "Hadamardian upper bound" $\max \left|\Delta^{-1}\right|$, the less sensitive system (4.1) is to errors. Moulton [15] chose instead the tetrahedron formed by the unit normals to the planes, whose volume is

$$
V^{\prime}=\frac{1}{n !} \frac{|\Delta|}{\max |\Delta|}
$$

This volume is soon computed once the determinant is known, and might accordingly be used rather than (5.4) to give some idea as to the system's sensitivity. When $V^{\prime}$ has its greatest possible value $(n !)^{-1}$, the vectors $\left(a_{11}, a_{12}, \cdots, a_{1 n}\right), \cdots,\left(a_{n 1}, a_{n 2}, \cdots, a_{n n}\right)$ are mutually orthogonal $[4, \mathrm{p}$. 31 ], whence so must be the hyperplanes (4.1); $V$ and $V^{\prime}$ attain their common maximum simultaneously.

6. Certain infinite systems. The space $\mathfrak{S}$ of Hilbert, in which $x$ has the denumerable infinity of components $x_{1}, x_{2}, \cdots$ and $\|x\|=\left(\sum_{i=1}^{\infty}\left|x_{i}\right|^{2}\right)^{1 / 2}$, is a Banach space. Consequently, if $y \in \mathfrak{S}$, the theory (a) of $\$ 1$ applies to an infinite linear system $(I+A) x=y$ :

$$
x_{i}+\sum_{j=1}^{\infty} a_{i j} x_{j}=y_{i}, \quad i=1,2, \cdots,
$$

provided that matrix $A=\left(a_{i j}\right)$ is bounded and

$$
M(A)<1 .
$$

(This was first established directly by E. Hilb $[17$, p. 92]. Discussions of the matrix calculus in $\mathfrak{W}$ are to be found in Hellinger and Toeplitz [8] and Riesz [17].) In fact, a bounded matrix $B=\left(b_{i j}\right)$ exists, defined by $I+B=(I+A)^{-1}$ $=I-A+A^{2}-A^{3}+\cdots$, in terms of which $x=(I+B) y$, or

$$
x_{i}=y_{i}+\sum_{j=1}^{\infty} b_{i j} y_{j}, \quad i=1,2, \cdots \text {. }
$$

Furthermore (1.10), $M(I+B) \leqq\{1-M(A)\}^{-1}$, or $m(I+A) \geqq 1-M(A)$; and 


$$
\|x\| \leqq\|y\| /\{1-M(A)\} .
$$

A condition which is sufficient (but by no means necessary) for boundedness of $A$ is that $\sum_{i=1}^{\infty} \sum_{j=1}^{\infty}\left|a_{i j}\right|^{2}=M^{*}(A)^{2}$ converge; then $M(A) \leqq M^{*}(A)$. A matrix $A$ for which $M^{*}(A)$ exists is completely continuous [17, p. 96].

If $\alpha=\left(\alpha_{i j}\right)$ is a bounded matrix with bound $M(\alpha)$ satisfying

$$
M(\alpha)<1-M(A),
$$

and if $\eta \in \mathfrak{S}$, the results of $\$ 2$ apply to the perturbed system

$$
x_{i}+\xi_{i}+\sum_{j=1}^{\infty}\left(a_{i j}+\alpha_{i j}\right)\left(x_{j}+\xi_{j}\right)=y_{i}+\eta_{i}, \quad i=1,2, \cdots,
$$

and (2.7) takes the form

$$
\|\xi\| \leqq\{\|\eta\|+M(\alpha)\|x\|\} /\{1-M(A)-M(\alpha)\},
$$

where of course $\|x\|$ is bounded by (6.4). From the foregoing can be found a limit for each $\left|\xi_{i}\right|$. For it follows from (6.1) and (6.6) that

$$
\xi_{i}=\eta_{i}-\sum_{j=1}^{\infty}\left(\alpha_{i j} x_{j}+a_{i j} \xi_{j}+\alpha_{i j} \xi_{j}\right), \quad i=1,2, \cdots,
$$

whence

$$
\begin{array}{r}
\left|\xi_{i}\right| \leqq\left|\eta_{i}\right|+\|\xi\|\left(\sum_{j=1}^{\infty}\left|a_{i j}\right|^{2}\right)^{1 / 2}+(\|x\|+\|\xi\|)\left(\sum_{j=1}^{\infty}\left|\alpha_{i j}\right|^{2}\right)^{1 / 2}, \\
i=1,2, \cdots .
\end{array}
$$

This, with (6.4) and (6.7), gives the desired bound. For bounded matrices $A$ and $\alpha$, the series $\sum_{j=1}^{\infty}\left|a_{i j}\right|^{2}$ and $\sum_{j=1}^{\infty}\left|\alpha_{i j}\right|^{2}$ converge $[17$, p. 47] for each $i=1,2, \cdots$.

7. Schmidt's criterion for existence of a bounded inverse. E. Schmidt $[19, \S 15]$ extended to an unbounded matrix $A=\left(a_{i j}\right)$ in $\mathfrak{S}$ a theorem of O. Toeplitz which asserts existence of a bounded inverse $A^{-1}$. While this inverse is not necessarily unique, the results of $\$ 2$ apply. Schmidt's premises are (1) that for each $i=1,2, \cdots, \sum_{j=1}^{\infty}\left|a_{i j}\right|^{2}$ converges, and (2) that every finite set of rows of $A$ is linearly independent. (The second is not an essential restriction.) Existence of the bounded inverse then depends on one further condition, which we shall now describe.

The system of equations to be solved is $A x=y$, or

$$
\sum_{j=1}^{\infty} a_{i j} x_{i}=y_{i}, \quad i=1,2, \cdots,
$$

where $y \in \mathfrak{S}$ and an $x$ in $\mathfrak{S}$ is sought. Schmidt replaces (7.1) by an equivalent "orthonormalized" system $B x=z$ : 


$$
\sum_{j=1}^{\infty} b_{i j} x_{i}=z_{i}, \quad i=1,2, \cdots,
$$

where the $b$ 's and $z$ 's are obtained from the $a$ 's and $y$ 's by equations of form

$$
b_{i j}=\sum_{k=1}^{i} g_{i k} a_{k j}, \quad z_{i}=\sum_{k=1}^{i} g_{i k} y_{k}
$$

in such a way that

$$
\sum_{k=1}^{\infty} b_{i k} b_{j k}=\delta_{i j}=\left\{\begin{array}{l}
0 \\
1
\end{array}\right.
$$

here $b_{j k}$ is the complex conjugate of $b_{j k}$. If $z \in \mathfrak{E}$, system (7.2) is solved by a vector $x$

$$
x_{i}=\sum_{j=1}^{\infty} b_{j i} z_{j}, \quad i=1,2, \cdots ;
$$

it follows from (7.4) that $\|x\|=\|z\|$; and Schmidt shows that (7.5) gives the solution of smallest norm, in case (7.1) has more than one solution. Thus the boundedness of the inverse $A^{-1}$ which yields solution (7.5) is made to depend on boundedness of the transformation $G=\left(g_{i j}\right)$ of (7.3).

If $\mu_{i j}=\bar{\mu}_{j i}=\sum_{k=1}^{\infty} a_{i k} \bar{a}_{j k},\left(\mu_{i j}\right)_{n}$, with $i, j \leqq n$, is a positive definite Hermitian matrix, and its characteristic roots $\lambda_{1}^{(n)} \leqq \lambda_{2}^{(n)} \leqq \cdots \leqq \lambda_{n}^{(n)}$ are all positive. Schmidt shows, in effect, that if $l=\lim \lambda_{1}^{(n)}$ is positive,

$$
M\left(A^{-1}\right) \leqq l^{-1 / 2} \text {. }
$$

Consequently, if in the perturbed system

$$
\sum_{j=1}^{\infty}\left(a_{i j}+\alpha_{i j}\right)\left(x_{i}+\xi_{i}\right)=y_{i}+\eta_{i}, \quad i=1,2, \cdots,
$$

matrix $\alpha=\left(\alpha_{i j}\right)$ is bounded and

$$
M(\alpha)<l^{1 / 2},
$$

the theorem of $\S 2$ can be applied and $\|\xi\| \leqq\{\|\eta\|+M(\alpha)\|x\|\} /\left\{l^{1 / 2}-M(\alpha)\right\}$, where $\|x\| \leqq\|y\| l^{-1 / 2}, x$ being the solution indicated in (7.5).

System (7.2) - and consequently (7.1) - has no other solution if [17, p. 70] the $b$ 's satisfy

$$
\sum_{k=1}^{\infty} b_{k i} b_{k j}=\left\{\begin{array}{l}
0 \\
1
\end{array}\right.
$$

if $i \neq j$, if $i=j$,

as well as (7.4). The condition is also necessary.

8. The method of segments. As applied to the system (6.1) in $\mathfrak{E}$, the method of segments consists in solving the (effectively algebraic) system 


$$
\begin{aligned}
x_{i}^{(n)}+\sum_{j=1}^{n} a_{i j} x_{j}^{(n)}=y_{i}, & i=1,2, \cdots, n, \\
x_{i}^{(n)}=0, & i=n+1, n+2, \cdots,
\end{aligned}
$$

in the hope that a solution $x^{(n)}$ of (8.1) is a "good enough" approximation to a solution $x$-if it exists - of (6.1). We assume that $A$ is bounded, but instead of requiring as in $\S 6$ that $M(A)<1$, we suppose that the determinant $D^{(n)}$ of the algebraic system (8.1a) is different from zero; thus (8.1) is uniquely solvable, and surely $x^{(n)} \in \mathfrak{S}$.

We write (8.1) in vector form as

$$
\left(I+A^{(n)}\right) x^{(n)}=y^{(n)} .
$$

Here $I$ is the identity matrix of $\mathfrak{S}$. Equation (6.1) can now be written as a perturbation of (8.1):

$$
\left(I+A^{(n)}+\alpha^{(n)}\right)\left(x^{(n)}+\xi^{(n)}\right)=y^{(n)}+\eta^{(n)} .
$$

Here $\alpha^{(n)}=A-A^{(n)}, \xi^{(n)}=\left(x_{i}-x_{i}^{(n)}\right), \eta^{(n)}=\left(0,0, \cdots, 0, y_{n+1}, y_{n+2}, \cdots\right)$. The infinite matrix $I+A^{(n)}$ has the (unique) inverse $I+B^{(n)}: B^{(n)}$ is the $n \times n$ square matrix $\left(b_{i j}^{(n)}\right)=\left(D_{j k}^{(n)} / D^{(n)}-\delta_{i j}\right)$, where $\delta_{i j}$ is the Kronecker delta and $D_{i j}^{(n)}$ is the cofactor of $\delta_{i, j}+a_{i j}$ in the determinant $D^{(n)}$ of (8.1a). By the Lagrange-Cauchy inequality and (1.4)

$$
M\left(I+B^{(n)}\right) \leqq 1+\left(\sum_{i=1}^{n} \sum_{j=1}^{n}\left|b_{i j}^{(n)}\right|^{2}\right)^{1 / 2}=1+Q_{n}
$$

Now if

$$
M\left(\alpha^{(n)}\right)=M\left(A-A^{(n)}\right)<\left(1+Q_{n}\right)^{-1},
$$

the theorem of $\$ 2$ applies; we can assert that (6.1) has a unique solution $x$ and that the error $\xi^{(n)}=x-x^{(n)}$ is bounded by

$$
\left\|\xi^{(n)}\right\| \leqq\left(1+Q_{n}\right)\left\{\left\|\eta^{(n)}\right\|+M\left(\alpha^{(n)}\right)\left\|x^{(n)}\right\|\right\} /\left\{1-M\left(\alpha^{(n)}\right)\left(1+Q_{n}\right)\right\} .
$$

It must be admitted that condition (8.4) is unfortunate. In the first place, one may expect that it will not of ten be easy to evaluate or even to estimate $M\left(A-A^{(n)}\right)$. In the second place, (8.4) requires that $M\left(A-A^{(n)}\right)$ be less than a bound which itself depends on $n$. If $A$ is completely continuous, $M\left(A-A^{(n)}\right) \rightarrow 0[17$, p. $113(\$ 77)]$; but whether the convergence is rapid enough to ensure (8.4) for some $n$ is another question $\left(^{5}\right)$. (In our discussion of a similar problem for integral equations $(\$ 11)$ it is possible to avoid the

(5) It is, of course, not essential to success of the method of segments that (8.4) be satisfied, as one may see in Riesz $[17, \S 77]$. The referee remarks that our first inequality (2.6) holds if $A$ is completely continuous and $(I-A)^{-1}$ exists; for then the sequence $M\left(I+B^{(n)}\right)$ is bounded and $M\left(T^{-1} \tau\right)=M\left(\left(I+B^{(n)}\right) \alpha^{(n)}\right) \rightarrow 0$. 
whole difficulty through explicit knowledge of $T^{-1}$ and $(T+\tau)^{-1}$.) A case in which $M\left(\alpha^{(n)}\right)$ may be estimated occurs when $M^{*}(A)^{2}=\sum_{i=1}^{\infty} \sum_{j=1}^{\infty}\left|a_{i j}\right|^{2}$ converges; then $M\left(\alpha^{(n)}\right) \leqq M^{*}\left(\alpha^{(n)}\right)$, and if $M^{*}\left(\alpha^{(n)}\right)<\left(1+Q_{n}\right)^{-1},(8.5)$ holds with $M\left(\alpha^{(n)}\right)$ replaced by $M^{*}\left(\alpha^{(n)}\right)$. If $M^{*}(A)<1$, a modification of the proof of (6.7) yields

$$
\left\|\xi^{(n)}\right\| \leqq\left\{\left\|\eta^{(n)}\right\|+M^{*}\left(\alpha^{(n)}\right)\left\|x^{(n)}\right\|\right\} /\left\{1-M^{*}(A)\right\} .
$$

In fact, (8.6) holds with $M$ instead of $M^{*}$, if $M(A)<1$.

Bounds for the components of $\xi^{(n)}$ can be found just as in $\S 6$. From combining (8.1) and (8.2) we see that

$$
\xi^{(n)}=\eta^{(n)}-\left(A-A^{(n)}\right) x^{(n)}-A \xi^{(n)},
$$

which breaks down into

$$
\begin{array}{lr}
\xi_{i}^{(n)}=-\sum_{j=1}^{\infty} a_{i j} \xi_{j}^{(n)}, & i=1,2, \cdots, n ; \\
\xi_{i}^{(n)}=y_{i}-\sum_{j=1}^{n} a_{i j} x_{j}^{(n)}-\sum_{j=1}^{\infty} a_{i j} \xi_{j}^{(n)}, & i=n+1, n+2, \cdots .
\end{array}
$$

From these, since $A$ is bounded,

$$
\begin{aligned}
\left|\xi_{i}^{(n)}\right| \leqq\left(\sum_{j=1}^{\infty}\left|a_{i j}\right|^{2}\right)^{1 / 2}\left\|\xi^{(n)}\right\|, \quad i=1,2, \cdots, n ; \\
\left|\xi_{i}^{(n)}\right| \leqq\left|y_{i}-\sum_{j=1}^{n} a_{i j} x_{j}^{(n)}\right|+\left(\sum_{j=1}^{\infty}\left|a_{i j}\right|^{2}\right)^{1 / 2}\left\|\xi^{(n)}\right\|, \\
i=n+1, n+2, \cdots .
\end{aligned}
$$

Of course, inequalities (8.7) represent no improvement over $\left|\xi_{i}^{(n)}\right| \leqq\left\|\xi^{(n)}\right\|$ if $\sum_{j=1}^{\infty}\left|a_{i j}\right|^{2} \geqq 1$.

9. A class of linear integral equations. In these last sections we shall deal only with real functions of real variables.

It is well known $[17$, p. 163] that a linear integral equation of Fredholm type and second kind

$$
x(s)+\int_{0}^{1} \mathrm{~K}(s, t) x(t) d t=y(s)
$$

is equivalent (under proper assumptions) to an infinite linear system of form (6.1). One might accordingly hope to solve (9.1) approximately by transforming it into such a system, and then applying the method of $\S 8$. In $\$ 11$ we shall limit the error for a similar but more direct scheme for solving (9.1). Our present purpose is to discuss the error-propagation question for (9.1) under assumptions strictly analogous to those of $\$ 6$. Our Banach space is the 
space $\mathcal{L}_{2}(0,1)$ of functions whose squares are integrable over the interval $(0,1)$ in the sense of Lebesgue; $\mathcal{L}_{2}(0,1)$ is equivalent to $\mathfrak{S}[2$, chap. 11 , Theorems 1 and 4]. Now all integrals are Lebesgue integrals; the norm is

$$
\|x\|=\left(\int_{0}^{1} x^{2}(s) d s\right)^{1 / 2}
$$

We assume that $y(s) \in \mathcal{L}_{2}(0,1)$; that a smallest positive constant $M(\mathrm{~K})$ exists satisfying

$$
\left\|\int_{0}^{1} \mathrm{~K}(s, t) z(t) d t\right\| \leqq M(\mathrm{~K})\|z\|
$$

whenever $z(s) \in \mathcal{L}_{2}(0,1)$; and that $M(\mathrm{~K})<1$. Then the hypotheses (a) of $\$ 1$ apply; (9.1) is uniquely solvable in $\mathcal{L}_{2}(0,1)$, and $\|x\| \leqq\|y\| /\{1-M(\mathrm{~K})\}$.

If in the perturbed equation

$$
x(s)+\xi(s)+\int_{0}^{1}\{\mathrm{~K}(s, t)+\kappa(s, t)\}\{x(t)+\xi(t)\} d t=y(s)+\eta(s),
$$

$\eta(s) \in \mathcal{L}_{2}(0,1)$ and $M(\kappa)<1-M(\mathrm{~K})$, a $\xi(s)$ in $\mathcal{L}_{2}(0,1)$ is uniquely determined and by (2.7)

$$
\|\xi\| \leqq\{\|\eta\|+M(\kappa)\|x\|\} /\{1-M(\mathrm{~K})-M(\kappa)\} .
$$

To this "global" bound can be added a "local" one, that is, a bound for $|\xi(s)|$, provided that $\mathrm{K}^{2}(s)=\int_{0}^{1} \mathrm{~K}^{2}(s, t) d t$ and $\kappa^{2}(s)=\int_{0}^{1} \kappa^{2}(s, t) d t$ exist. For from (9.1) and (9.3)

$$
\xi(s)=\eta(s)-\int_{0}^{1} \kappa(s, t) x(t) d t-\int_{0}^{1}\{\mathrm{~K}(s, t)+\kappa(s, t)\} \xi(t) d t .
$$

whence by the Bouniakowsky-Schwarz inequality

$$
|\xi(s)| \leqq|\eta(s)|+\mathrm{K}(s)\|\xi\|+\kappa(s)(\|x\|+\|\xi\|) .
$$

The (linear) space of functions continuous on the closed interval $(0,1)$ with norm (9.2) is not complete. If $\mathrm{K}^{2}(s, t)$ is integrable (Riemann) over the square $0 \leqq s \leqq 1,0 \leqq t \leqq 1$, the transformation $\mathrm{K} x \equiv \int_{0}^{1} \mathrm{~K}(s, t) x(t) d t$ is completely continuous [2, p. 99]; and if furthermore

$$
M^{*}(\mathrm{~K})=\left(\int_{0}^{1} \int_{0}^{1} \mathrm{~K}^{2}(s, t) d s d t\right)^{1 / 2}<1
$$

hypotheses (b) of $\S 1$ are fulfilled. Hence if $y(s)$ is continuous, (9.1) has a unique continuous solution. If also $M^{*}(\kappa)<1-M^{*}(\mathrm{~K})$, inequality (9.5) holds with $M$ replaced by $M^{*}$; (9.6) likewise follows.

10. The Fredholm formulas. Fredholm showed that if the kernel $\mathbf{K}(s, t)$ 
of integral equation

$$
x(s)-\lambda \int_{0}^{1} \mathrm{~K}(s, t) x(t) d t=y(s)
$$

is continuous on the closed square $0 \leqq s, t \leqq 1$, certain power series in $\lambda, D(\lambda)$ and $D(s, t ; \lambda)[4$, p. 123$]$ converge for all values of $\lambda$. If $D(\lambda) \neq 0, \mathrm{~K}^{*}(s, t ; \lambda)$ $=-D(s, t ; \lambda) / D(\lambda)$ is a resolvent kernel for (10.1), in terms of which the unique continuous solution of (10.1) can be presented as

$$
x(s)=y(s)+\lambda \int_{0}^{1} \mathrm{~K}^{*}(s, t ; \lambda) y(t) d t
$$

if $y(s)$ is continuous on $(0,1)$. Briefly, the inverse of $T \equiv I-\lambda \mathrm{K}$ is $T^{-1} \equiv I+\lambda \mathrm{K}^{*}$. We take for our linear vector space the set of functions continuous on $0 \leqq s \leqq 1$, with norm

$$
\|x\|=\max _{(s)}|x(s)|
$$

Convergence is now uniform convergence on $(0,1)$, so the space is complete, hence a Banach space. From (10.2)

$$
\|x\| \leqq\|y\|\left\{1+|\lambda| M\left(\mathrm{~K}^{*}\right)\right\}
$$

where

$$
M\left(\mathrm{~K}^{*}\right)=\max _{(s)}\left\{\max _{(s)}\left|\int_{0}^{1} \mathrm{~K}^{*}(s, t ; \lambda) z(t) d t\right| / \max _{(s)}|z(s)|\right\} .
$$

It is clear from (10.2) that

$$
M\left(\mathrm{~K}^{*}\right) \leqq \max _{(s)} \int_{0}^{1}\left|\mathrm{~K}^{*}(s, t ; \lambda)\right| d t \leqq \max _{(s, t)}\left|\mathrm{K}^{*}(s, t ; \lambda)\right| .
$$

If $\lambda$ is such that $|D(\lambda)| \geqq \epsilon_{\lambda}>0$, and if $\overline{\mathrm{K}}$ is an upper bound for $\mathrm{K}(s, t)$ on the square $0 \leqq s, t \leqq 1$, the well known Fredholm bound for $M\left(\mathrm{~K}^{*}\right)$ is

$$
M\left(\mathrm{~K}^{*}\right) \leqq \max _{(s, t)}\left|\mathrm{K}^{*}(s, t ; \lambda)\right| \leqq \frac{\overline{\mathrm{K}}}{\epsilon_{\lambda}} \Omega^{\prime}(|\lambda| \overline{\mathrm{K}}),
$$

where $\Omega(z)=\sum_{n=0}^{\infty} n^{n / 2} z^{n} / n !$. Thus

$$
M\left(T^{-1}\right) \leqq 1+\frac{\overline{\mathrm{K}}}{\epsilon_{\lambda}} \Omega^{\prime}(|\lambda| \overline{\mathbf{K}}) .
$$

$\Omega(z)$ has been tabulated by Tricomi $\left[21,2^{\circ}\right.$, p. 28$]$ from $z=0.00$ to $z=1.00$ at intervals of 0.05 , and from these $\Omega^{\prime}(z)$ can be approximated. Tricomi also shows that $(1+z) e^{e z^{2} / 2}$ is a majorant of $\Omega(z)$, so that $\Omega^{\prime}(z) \leqq\left(1+e z+e z^{2}\right) e^{e z^{2} / 2}$. 
For convenience, we now suppose $\lambda=-1$ and write $\epsilon$ for $\epsilon_{-1}$. Equations (9.1) and (9.3) will determine a continuous $\xi(s)$, under present conditions, if $\kappa(s, t)$ is continuous and if

$$
M(\kappa)<\left\{1+\frac{\overline{\mathbf{K}}}{\epsilon} \Omega^{\prime}(\overline{\mathbf{K}})\right\}^{-1} ;
$$

it is sufficient, though not necessary, to have

$$
\bar{\kappa}=\max _{(s, t)}|\kappa(s, t)|<\left\{1+\frac{\overline{\mathbf{K}}}{\epsilon} \Omega^{\prime}(\overline{\mathbf{K}})\right\}^{-1} .
$$

since $M(\kappa) \leqq \bar{\kappa}$. Then (2.7) applies to limit $\|\xi\|=\max |\xi(s)|$ :

$$
\|\xi\| \leqq\{\|\eta\|+M(\kappa)\|x\|\} /\left\{\left[1+\frac{\overline{\mathbf{K}}}{\epsilon} \Omega^{\prime}(\overline{\mathrm{K}})\right]^{-1}-M(\kappa)\right\} .
$$

The problem of this section was solved rather differently by Tricomi [21]. Related investigations are due to Mrs. Adams [1].

Our methods permit us to go somewhat further, in allowing $\alpha(s, t)$ to be discontinuous to a certain degree. We retain continuity of $\mathrm{K}(s, t)$ and of $y(s)$, but ask of $\kappa(s, t)$ only that the Riemann integrals

$$
M^{*}(\kappa)^{2}=\int_{0}^{1} \int_{0}^{1} \kappa^{2}(s, t) d s d t, \quad \kappa^{2}(s)=\int_{0}^{1} \kappa^{2}(s, t) d t
$$

exist. Our vector space still consists of functions continuous on $0 \leqq s \leqq 1$, but norm (10.3) is replaced by norm (9.2). This space is not complete. However, kernel $\kappa(s, t)$ defines a completely continuous transformation, so hypotheses (b) of $\$ 2$ are in force; the estimate of $M\left(\mathrm{~K}^{*}\right)$ is still valid, so that inequality (10.6) still holds subject to (10.5), provided that $M(\kappa)$ is replaced by $M^{*}(\kappa)$, and by norm is meant (9.2).

A local bound for $|\xi(s)|$ can be found exactly as in $\S 9$.

11. The method of "kernel-splitting": Fredholm equations of second kind. Tricomi's investigation [21] was motivated by the need for a limitation of the error incurred when kernel $\mathrm{K}(s, t)$ in (9.1) is replaced by an approximation of form $\mathrm{K}_{n}(s, t)=\sum_{j=1}^{j=n} \alpha_{j}(s) \beta_{j}(t)$. With a kernel of this type, the integral equation reduces to a system of $n$ linear algebraic equations in $n$ unknowns. Tricomi ascribes the process to Goursat and Pincherle; Goursat [7] used it to derive the Fredholm formulas for a continuous $\mathbf{K}(s, t)$, and Lebesgue [8] extended his results. Here we shall follow the alternative development of Schmidt [18], who derived the Fredholm theorems (concerning existence and multiplicity of solutions) but not the Fredholm formulas; the formulas he actually found lead to a direct and easy solution of the problem at hand, as will be seen. 
Schmidt supposes functions $\alpha_{1}(s), \cdots, \alpha_{n}(s), \beta_{1}(t), \cdots, \beta_{n}(t)$ to be known such that, if $\kappa_{n}(s, t)=\mathbf{K}(s, t)-\sum_{j=1}^{j=n} \alpha_{j}(s) \beta_{j}(t)$, there is a positive $\epsilon$ satisfying

$$
M^{*}\left(\kappa_{n}\right)^{2}=\int_{0}^{1} \int_{0}^{1} \kappa_{n}^{2}(s, t) d s d t \leqq \epsilon^{2}<1 .
$$

The approximating integral equation

$$
x_{n}(s)+\int_{0}^{1} \sum_{j=1}^{n} \alpha_{j}(s) \beta_{j}(t) x_{n}(t) d t=y(s),
$$

obtained by replacing $\mathrm{K}(s, t)$ in (9.1) by its bilinear-sum approximant, reduces to the algebraic system for $\rho_{1}, \cdots, \rho_{n}$

$$
\rho_{i}+\sum_{j=1}^{n} \rho_{j} \int_{0}^{1} \alpha_{j}(s) \beta_{i}(s) d s=\int_{0}^{1} y(s) \beta_{i}(s) d s, \quad i=1,2, \cdots, n,
$$

where

$$
\rho_{i}=\int_{0}^{1} \beta_{i}(s) x_{n}(s) d s
$$

we assume that the determinant $\Delta$ of (11.3) does not vanish. Then, from (11.2),

$$
x_{n}(s)=y(s)-\sum_{j=1}^{n} \rho_{j} \alpha_{j}(s) .
$$

If $A_{i j}$ is the cofactor of the element in the $i$ th row and $j$ th column of $\Delta$,

$$
\rho_{i}=\frac{1}{\Delta} \sum_{j=1}^{n} A_{j i} \int_{0}^{1} y(s) \beta_{j}(s) d s,
$$

so by (11.5)

$$
x_{n}(s)=y(s)-\frac{1}{\Delta} \sum_{i=1}^{n} \sum_{j=1}^{n} A_{j i} \alpha_{i}(s) \int_{0}^{1} y(t) \beta_{j}(t) d t .
$$

Schmidt shows $[18$, p. 171] that the exact solution $x(s)$ of $(9.1)$ is

$$
\begin{aligned}
x(s)=y(s)- & \int_{0}^{1}\left\{\kappa_{n}^{*}(s, t)+\frac{1}{\Delta} \sum_{i=1}^{n} \sum_{j=1}^{n} A_{i j}\left(\alpha_{j}(s)\right.\right. \\
& \left.\left.+\int_{0}^{1} \kappa_{n}^{*}(s, r) \alpha_{j}(r) d r\right)\left(\beta_{i}(t)+\int_{0}^{1} \kappa_{n}^{*}(r, t) \beta_{i}(r) d r\right)\right\} y(t) d t,
\end{aligned}
$$

where $\kappa_{n}^{*}=\sum_{j=0}^{\infty}\left(-\kappa_{n}\right)^{j}$ is the resolvent kernel associated with $\kappa_{n}$. With norm 
(9.2), $M\left(\kappa_{n}^{*}\right) \leqq \epsilon /(1-\epsilon)$; and the Schwarz inequality applied to the difference $x-x_{n}$ gives

$$
\left\|x-x_{n}\right\|
$$

$$
\leqq \frac{\epsilon}{1-\epsilon}\left\{1+\frac{1}{|\Delta|}\left(\sum_{i=1}^{n} \sum_{j=1}^{n} A_{i j}^{2}\left\|\alpha_{j}\right\|^{2}\left\|\beta_{i}\right\|^{2}\right)^{1 / 2}\left(2+\frac{\epsilon}{1-\epsilon}\right)\right\}\|y\| .
$$

This inequality, being derived independently of our general theory, places no restriction on $\epsilon$ other than (11.1). A local bound for $\left|x(s)-x_{n}(s)\right|$ can be written down as in $\$ 9$.

In the first paper of the series of which [18] is the second, Schmidt determines best expansion functions $\alpha_{i}(s), \beta_{i}(t)$. Bateman [3] has suggested a scheme for constructing certain polynomial $\alpha$ 's and $\beta$ 's which, while not necessarily "best," promise greater usefulness in practise.

12. Linear integral equations of first kind and Fredholm type. The theory of equation

$$
\int_{0}^{1} \mathrm{~K}(s, t) x(t) d t=y(s)
$$

is much less complete than that of (9.1). A well known necessary and sufficient condition for existence of a solution in $\mathcal{L}_{2}(0,1)$ is due to Picard $[11$, p. 99].

Here we shall present an error-limit for a method of solving (12.1) which is similar to that of $\S 11$, though less flexible. Existence of $\|y\|$ in the sense of (9.2) is assumed. We further assume knowledge of two sets of functions, each consisting of $n$ functions which are linearly independent and orthonormal on $(0,1)$, say

$$
\alpha_{1}(s), \cdots, \alpha_{n}(s) ; \quad \beta_{1}(t), \cdots, \beta_{n}(t) ;
$$

and $n$ nonzero constants $k_{1}, \cdots, k_{n}$, such that if

$$
\begin{aligned}
\kappa_{n}(s, t) & =\mathrm{K}(s, t)-\mathrm{K}_{n}(s, t) \\
& =\mathrm{K}(s, t)-\sum_{i=1}^{n} k_{i} \alpha_{i}(s) \beta_{i}(t),
\end{aligned}
$$

then

$$
\int_{0}^{1} \int_{0}^{1} \kappa_{n}^{2}(s, t) d s d t \leqq \epsilon^{2} ;
$$

this (positive) $\epsilon$ will later be restricted by (12.8). Then equation

$$
\int_{0}^{1} \mathrm{~K}_{n}(s, t) x_{n}(t) d t=y_{n}(s) .
$$

where 


$$
y_{n}(s)=\sum_{i=1}^{n} \pi_{i} \alpha_{i}(s), \quad \pi_{i}=\int_{0}^{1} y(t) \alpha_{i}(t) d t,
$$

can be solved for $x_{n}(s)$. For (12.4) can be rewritten as

$$
\sum_{i=1}^{n}\left(k_{i} \rho_{i}-\pi_{i}\right) \alpha_{i}(s)=0
$$

where

$$
\rho_{i}=\int_{0}^{1} x_{n}(t) \beta_{i}(t) d t
$$

since the $\alpha$ 's are linearly independent and $k_{i} \neq 0, \rho_{i}=\pi_{i} / k_{i}, i=1,2, \cdots, n$. Hence one solution of (12.4) is given by

$$
x_{n}(s)=\sum_{i=1}^{n} \rho_{i} \beta_{i}(s)=\sum_{i=1}^{n} \beta_{i}(s) \int_{0}^{1} y(t) \alpha_{i}(t) d t / k_{i} .
$$

By the Schwarz inequality, with quadratic norm (9.2),

$$
\left\|x_{n}\right\| \leqq\left(\sum_{i=1}^{n} k_{i}^{-2}\right)^{1 / 2}\|y\| .
$$

If $\eta_{n}=y-y_{n}$,

$$
\left\|\eta_{n}\right\|^{2}=\|y\|^{2}-\sum_{i=1}^{n} \pi_{i}^{2}
$$

and if $\|y\|>0$,

$$
\left\|x_{n}\right\| \leqq\left(1-\frac{\left\|\eta_{n}\right\|}{\|y\|}\right)^{-1}\left(\sum_{i=1}^{n} k_{i}^{-2}\right)^{1 / 2}\left\|y_{n}\right\| .
$$

We may think of (12.4) as $T_{n} x_{n}=y_{n}$; then

$$
M\left(T_{n}^{-1}\right) \leqq\left(1-\frac{\left\|\eta_{n}\right\|}{\|y\|}\right)^{-1}\left(\sum_{i=1}^{n} k_{i}^{-2}\right)^{1 / 2}
$$

Inequality (2.7) will consequently apply in the form

(12.7) $\left\|x-x_{n}\right\| \leqq\left\{\left\|\eta_{n}\right\|+\epsilon\left\|x_{n}\right\|\right\} /\left\{\left(1-\left\|\eta_{n}\right\| /\|y\|\right)\left(\sum_{i=1}^{n} k_{i}^{-2}\right)^{-1 / 2}-\epsilon\right\}$

provided that $\left\|\eta_{n}\right\|<\|y\|$ and

$$
\epsilon<\left(1-\left\|\eta_{n}\right\| /\|y\|\right)\left(\sum_{i=1}^{n} k_{i}^{-2}\right)^{-1 / 2} .
$$

We obtain no local bound for $\left|x(s)-x_{n}(s)\right|$. 


\section{REFERENCES}

1. Rachel B. Adams, On the approximate solution of Fredholm's homogeneous integral equation, Amer. J. Math. vol. 51 (1929) pp. 139-148.

2. Stefan Banach, Theorie des operations lineares, Warsaw, 1932.

3. Harry Bateman, On the numerical solution of linear integral equations, Proc. Roy. Soc. London Ser. A vol. 100 (1922) pp. 441-449.

4. Richard Courant and David Hilbert, Methoden der mathematischen Physik, vol. 1, 2d ed., Berlin, 1931.

5. H. T. Davis, The theory of linear operators, Bloomington, 1936.

6. I. M. H. Etherington, On errors in determinants, Proceedings of the Edinburgh Mathematical Society (2) vol. 3 (1932) pp. 107-117.

7. Emile Goursat, Sur un cas elementaire de l'équation de Fredholm, Bull. Soc. Math. France vol. 35 (1907) pp. 163-173.

8. Ernst Hellinger and Otto Toeplitz, Grundlagen fibr eine Theorie der unendlichen Matrizen, Math. Ann. 69 (1910) pp. 289-330.

9. Harold Hotelling, Some new methods in matrix calculation, Ann. Math. Statist. vol. 14 (1943) pp. 1-34.

10. - Further points on matrix calculation and simultaneous equations, Ann. Math. Statist. vol. 14 (1943) pp. 440-441.

11. Trajan Lalesco, Introduction da la theorie des equations intégrales, Paris, 1912.

12. Henri Lebesgue, Sur la methode de M. Goursat pour la résolution de l'équation de Fredholm, Bull. Soc. Math. France vol. 36 (1908) pp. 3-19.

13. A. T. Lonseth, Systems of linear equations with coefficients subject to error, Ann. Math. Statist. vol. 13 (1942) pp. 332-337.

14. - On relative errors in systems of linear equations, Ann. Math. Statist. vol. 15 (1944) pp. 323-325.

15. F. R. Moulton, On the solutions of linear equations having small determinants, Amer. Math. Monthly vol. 20 (1913) pp. 242-249.

16. J. W. S. Rayleigh, The theory of sound, vol. 2, 2d ed., London, 1896.

17. Frédéric Riesz, Les systèmes d'équations linéaires d une infinité d'inconnues, Paris, 1913.

18. Erhard Schmidt, Zur Theorie der linearen und nichtlinearen Integralgleichungen (II), Math. Ann. vol. 64 (1907) pp. 161-174.

19. - UUber die Auflösung linearer Gleichungen mit unendlich vielen Unbekannten, Rend. Circ. Mat. Palermo vol. 25 (1908) pp. 53-77.

20. J. L. Synge, $A$ geometrical interpretation of the relaxation method, Quarterly Journal of Applied Mathematics vol. 2 (1944) pp. 87-89.

21. Francesco Tricomi, Sulla risoluzione numerica delle equazioni integrali di Fredholm, Atti della Reale Accademia Nazionale dei Lincei, Rendiconti, Classe di Scienze Fisiche, Matematiche, e Naturali (5) vol. 33 (1924) $1^{\circ}$ sem. pp. 483-486, $2^{\circ}$ sem. pp. 26-30.

\section{NORTHWESTERN UNIVERSITY,} Evanston, ILl. 